\title{
FAMILIES OF TRIPLES WITH HIGH MINIMUM DEGREE ARE HAMILTONIAN
}

\author{
VOJTECH RÖDL ${ }^{1}$ \\ Emory University \\ Atlanta, GA, USA \\ e-mail: rodl@mathcs.emory.edu \\ AND \\ Andrzej Ruciński ${ }^{2}$ \\ A. Mickiewicz University \\ Poznań, Poland \\ e-mail: rucinski@amu.edu.pl
}

\begin{abstract}
In this paper we show that every family of triples, that is, a 3-uniform hypergraph, with minimum degree at least $\left(\frac{5-\sqrt{5}}{3}+\gamma\right)\left(\begin{array}{c}n-1 \\ 2\end{array}\right)$ contains a tight Hamiltonian cycle.

Keywords: 3-uniform hypergraph, Hamilton cycle, minimum vertex degree.
\end{abstract}

2010 Mathematics Subject Classification: 05D05, 05C65.

\section{REFERENCES}

[1] R. Aharoni, A. Georgakopoulos and P. Sprüssel, Perfect matchings in r-partite rgraphs, European J. Combin. 30 (2009) 39-42. doi:10.1016/j.ejc.2008.02.011

[2] E. Buss, H. Hàn and M. Schacht, Minimum vertex degree conditions for loose Hamilton cycles in 3-uniform hypergraphs, J. Combin. Theory (B), to appear.

\footnotetext{
${ }^{1}$ Research supported by NSF grants DMS 080070 and DMS-1102086.

${ }^{2}$ Research supported by the Polish NSC grant N201 604940 and the NSF grant DMS-1102086. Part of research performed at Emory University, Atlanta.
} 
[3] R. Glebov, Y. Person and W. Weps, On extremal hypergraphs for hamiltonian cycles, European J. Combin. 33 (2012) 544-555 (An extended abstract has appeared in the Proceedings of EuroComb 2011). doi:10.1016/j.ejc.2011.10.003

[4] H. Hàn, Y. Person and M. Schacht, On perfect matchings in uniform hypergraphs with large minimum vertex degree, SIAM J. Discrete Math. 23 (2009) 732-748. doi:10.1137/080729657

[5] H. Hàn and M. Schacht, Dirac-type results for loose Hamilton cycles in uniform hypergraphs, J. Combin. Theory (B) 100 (2010) 332-346. doi:10.1016/j.jctb.2009.10.002

[6] S. Janson, T. Łuczak and A. Ruciński, Random Graphs (John Wiley and Sons, New York, 2000). doi:10.1002/9781118032718

[7] G.Y. Katona and H.A. Kierstead, Hamiltonian chains in hypergraphs, J. Graph Theory 30 (1999) 205-212. doi:10.1002/(SICI)1097-0118(199903)30:3〈205::AID-JGT5〉3.0.CO;2-O

[8] P. Keevash, D. Kühn, R. Mycroft and D. Osthus, Loose Hamilton cycles in hypergraphs, Discrete Math. 311 (2011) 544-559. doi:10.1016/j.disc.2010.11.013

[9] I. Khan, Perfect matching in 3-uniform hypergraphs with large vertex degree, SIAM J. Discrete Math. 27 (2013) 1021-1039. doi: $10.1137 / 10080796 \mathrm{X}$

[10] D. Kühn, R. Mycroft and D. Osthus, Hamilton l-cycles in uniform hypergraphs, J. Combin. Theory (A) 117 (2010) 910-927. doi:10.1016/j.jcta.2010.02.010

[11] D. Kühn and D. Osthus, Matchings in hypergraphs of large minimum degree, J. Graph Theory 51 (2006) 269-280. doi:10.1002/jgt.20139

[12] D. Kühn and D. Osthus, Loose Hamilton cycles in 3-uniform hypergraphs of high minimum degree, J. Combin. Theory (B) 96 (2006) 767-821. doi:10.1016/j.jctb.2006.02.004

[13] D. Kühn, D. Osthus and A. Treglown, Matchings in 3-uniform hypergraphs, J. Combin. Theory (B) 103 (2013) 291-305. doi:10.1016/j.jctb.2012.11.005

[14] O. Pikhurko, Perfect matchings and $K_{4}^{3}$-tilings in hypergraphs of large codegree, Graphs Combin. 24 (2008) 391-404. doi:10.1007/s00373-008-0787-7

[15] V. Rödl and A. Ruciński, Dirac-type questions for hypergraphs - a survey (or more problems for Endre to solve), An Irregular Mind (Szemerédi is 70), Bolyai Soc. Math. Stud. 21 (2010) 561-590. 
[16] V. Rödl, A. Ruciński and E. Szemerédi, A Dirac-type theorem for 3-uniform hypergraphs, Combin. Probab. Comput. 15 (2006) 229-251. doi:10.1017/S0963548305007042

[17] V. Rödl, A. Ruciński and E. Szemerédi, Perfect matchings in uniform hypergraphs with large minimum degree, European. J. Combin. 27 (2006) 1333-1349. doi:10.1016/j.ejc.2006.05.008

[18] V. Rödl, A. Ruciński and E. Szemerédi, An approximate Dirac-type theorem for $k$ uniform hypergraphs, Combinatorica 28 (2008) 229-260. doi: $10.1007 /$ s00493-008-2295-z

[19] V. Rödl, A. Ruciński and E. Szemerédi, Perfect matchings in large uniform hypergraphs with large minimum collective degree, J. Combin. Theory (A) 116 (2009) 613-636. doi:10.1016/j.jcta.2008.10.002

[20] V. Rödl, A. Ruciński and E. Szemerédi, Dirac-type conditions for hamiltonian paths and cycles in 3-uniform hypergraphs, Adv. Math. 227 (2011) 1225-1299. doi:10.1016/j.aim.2011.03.007

[21] V. Rödl, A. Ruciński, M. Schacht and E. Szemerédi, A note on perfect matchings in uniform hypergraphs with large minimum collective degree, Comment. Math. Univ. Carolin. 49 (2008) 633-636.

Received 9 November 2012

Revised 25 April 2013

Accepted 25 April 2013 